\title{
Dynamic Excimer (DYNEX) Imaging of Lipid Droplets
}

\author{
M. Carmen Gonzalez-Garcia, Carmen Salto-Giron, Pilar Herrero-Foncubierta, Tomás Peña-Ruiz, \\ Maria Dolores Giron-Gonzalez, Rafael Salto-Gonzalez, Angel Perez-Lara, Amparo Navarro, \\ Emilio Garcia-Fernandez,* and Angel Orte*
}

Cite This: https://doi.org/10.1021/acssensors.1c01206

Read Online

ABSTRACT: Unraveling cellular physiological processes via luminescent probes that target specific cellular microenvironments is quite challenging due to the uneven distribution of probes. Herein, we designed a new dynamic excimer (DYNEX) imaging method that involves the sensitive detection of nanosecond-scale dynamic molecular contacts of a fluorescent acridone derivative and reveals the cell microenvironment polarity. Using our method, we specifically tracked cell lipid droplets in fibroblast colon carcinoma cells. These organelles play a central role in metabolic pathways, acting as energy reservoirs in regulatory processes. DYNEX imaging provides the inner polarity of cell lipid droplets, which can be related to lipid contents and metabolic dysfunctions. This new methodology will inspire development of novel multidimensional fluorescent sensors that are able to provide target-specific and orthogonal information at the nanosecond scale.

KEYWORDS: cell microenvironment, excimer imaging, fluorescent probes, imaging agents, lipids, multiparametric microscopy

$\mathrm{F}$ luorescence microscopy is an extensively used platform to characterize membrane and membraneless organelles due to its high sensitivity, accuracy, and noninvasiveness. Specific chemically driven targeting of these cell organelles is based on their cellular microenvironment, which is defined by different parameters, such as macromolecules, salt types and concentration, temperature, $\mathrm{pH}$, viscosity, and polarity. ${ }^{1}$ Thus, the development of new sensors to monitor the dynamics of these parameters is key to understanding many cellular physiological processes and diseases in biology. In this work, we employed a chemically simple, fluorescent probe, $N$-(3-(benzyloxy)propyl)2-methoxy-acridin-9-one (1, Figure 1$)$, to study the polarity of lipid droplets. These organelles act as lipid storage units and are involved in prominent trafficking pathways, dynamically interacting with practically all subcellular structures. Importantly, dysregulation of lipid droplets has been associated with several diseases, such as lipodystrophies, fatty liver disease, or atherosclerosis. ${ }^{2,3}$ Therefore, lipid droplets are interesting target organelles in the study of altered metabolic states, such as highly proliferative tumor cells. ${ }^{2}$ Additionally, microenvironment polarity is an intrinsically complex parameter involving the medium acting as a continuous phase and specific molecular interactions of dispersion forces, dipole-dipole interactions, and hydrogen bonds, affecting enzymatic activity, membrane transport, and cellular proliferation. ${ }^{4}$ Thus, microenvironment polarity inside lipid droplets reveals different cellular metabolic states, either physiological or pathological. ${ }^{5-9}$

In a previous work, we designed a robust and quantitative methodology to obtain intracellular polarity in terms of the $\mathrm{E}_{\mathrm{T}} 30$ parameter by multiparametric microscopy and simultaneously employing two orthogonal imaging approaches, namely, time- gated ratiometric and fluorescence lifetime imaging. ${ }^{10} \mathrm{The}_{\mathrm{T}} 30$ parameter, defined as the difference in energy between the excited state and the ground state of the pyridinium- $N$ phenolate of betaine, has an advantage over other polarity parameters combining general solvent effects and hydrogenbonding interactions. ${ }^{11}$ Our method employs acridone derivatives as fluorescent reporters because of their excellent photophysical properties, which include long lifetimes (7-22 $\mathrm{ns})$, high quantum yields $(>0.6)$, and great $\mathrm{pH}$ stability at physiological levels. Importantly, the long fluorescence lifetime of these probes overcomes the problems of interferences caused by cellular autofluorescence by applying suitable time gates to filter out short-lived, interfering photons after excitation. ${ }^{12}$ Moreover, the subcellular localization of acridones can be finely tailored by incorporating directing molecules into the structural groups that do not alter the photophysical properties or performance of the dyes. ${ }^{13}$ Another interesting and distinctive feature of these acridone derivatives is their excited-state dynamics, including the excited-state formation of an excimer in solution. ${ }^{14}$

Taking advantage of this property, herein, we propose a new approach to organelle-specific tracking using a new layer of information based on the kinetics of the acridone excited state.

Received: June 8, 2021 
<smiles>COC1=CC2C(=O)c3ccccc3N(CCCOCc3ccccc3)C2C=C1</smiles>

1

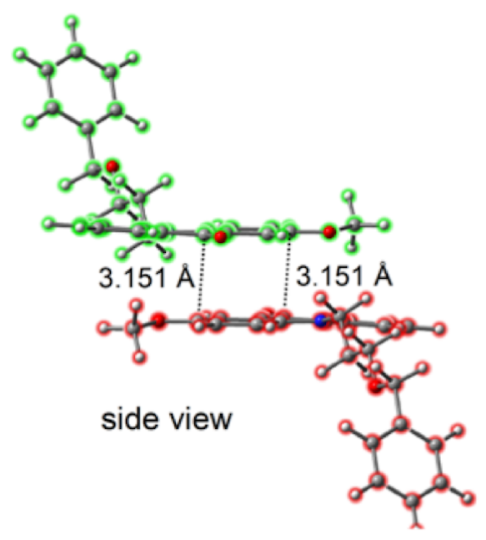

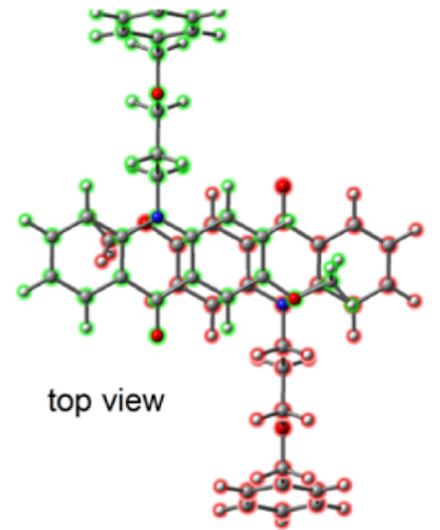
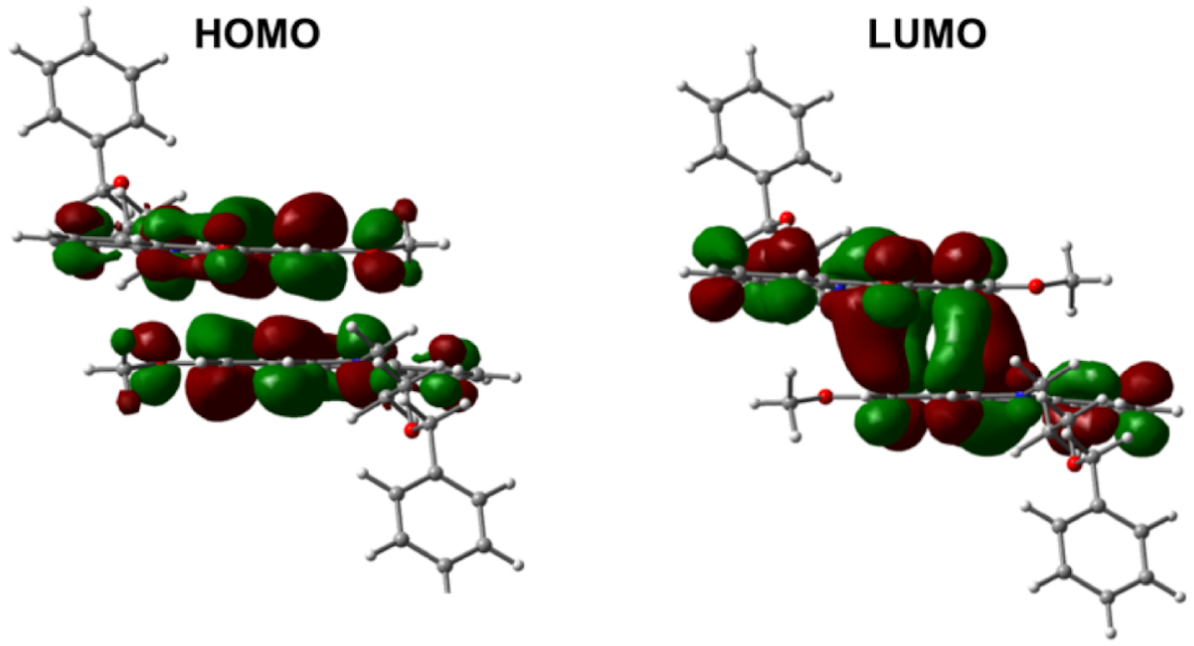

Figure 1. TD-CAM-B3LYP-D3/6-31+G** calculation results of the molecular geometry and HOMO and LUMO (isocontour plots 0.02 au) of an antiparallel excimer of acridone $\mathbf{1}$ in water solution.

This segmentation approach is subsequently combined with a previously validated method to probe polarity microenvironments, ${ }^{10}$ resulting in a novel imaging approach. The novelty of this DYNamic EXcimer (DYNEX) imaging method relies on the sensitive and specific detection of the dynamic excited-state formation of excimers of acridone 1 inside live cells. We used this feature as a tool to clearly identify, track, and specifically segment lipid droplets, the organelles in which we detected such dynamics. This adds a new and complementary layer of information to conventional fluorescence intensity and lifetime imaging.

\section{RESULTS AND DISCUSSION}

Excited-State Dynamics. To develop the DYNEX method, we first thoroughly investigated the excimer formation dynamics of compound $\mathbf{1}$ in different solvents using a combination of spectroscopic and computational tools. Time-dependent density functional theory (TD-DFT) calculations, using the TD-CAM-B3LYP/6-31+G** level of theory (see the Supporting Information, SI, for details), were performed to understand excimer formation at a molecular level, taking into account dispersion interactions in the dimer by including an empirical dispersion correction (GD3). ${ }^{15}$ Since 1 has a bulky $N$ substituent in the acridone core, we also studied unsubstituted 4-methoxyacridone and other simple derivatives (Chart S1 in the Supporting Information, SI). Modeling of such simpler molecular systems helped to optimize the different config- urations of the dimers in excited states as well as to investigate whether the hydrophobicity of the benzyl radical in $\mathbf{1}$ is involved in the excimer stability. Four different excimer arrangements were found in both water and cyclohexane (Figure S1 and Table S1 in the SI), with the structure with antiparallel packing being the most stable. Importantly, the lowest unoccupied molecular orbital (LUMO) wavefunction presented a clear overlap delocalized between the two acridone monomers, while in the highest occupied molecular orbital (HOMO), the molecular orbitals were isolated on the individual molecules (Figure 1). This result is a clear indication of the formation of excited-state $\pi-\pi$ interactions and, hence, of excimer formation. Some interatomic distances between carbon atoms are shown in Figure 1, which are within the interval of previous studies for aromatic compounds, $3.1-3.4 \AA^{16}$

We also investigated excimer formation using absorption, fluorescence (Figure S2), and time-resolved emission spectroscopy (TRES). TRES is an extremely valuable tool that allows following the nanosecond-scale temporal evolution of the emission spectrum, identifying excited-state dynamics, such as solvent relaxation or excited-state reactions. ${ }^{14,17,18}$ Although the formation of excimers of 4-methoxyacridone derivatives in aqueous solution has been previously established, ${ }^{14}$ it was not confirmed for acridone $\mathbf{1}$ due to its low solubility in water. This is an important feature since the formation of excimers will be favored in nonaqueous environments. Therefore, we first studied the excited-state dynamics of acridone $\mathbf{1}$ in acetonitrile 
$\left(\mathrm{E}_{\mathrm{T}} 30=45.6 \mathrm{kcal} \mathrm{mol}^{-1}\right)$ and methanol $\left(\mathrm{E}_{\mathrm{T}} 30=55.4 \mathrm{kcal}\right.$ $\left.\mathrm{mol}^{-1}\right)$. In acetonitrile, acridone 1 presented two decay times: a long lifetime of $10.26 \mathrm{~ns}$ and a short lifetime of $0.98 \mathrm{~ns}$. The TRES spectra (Figure 2A) exhibited an isoemissive point at 467

A)
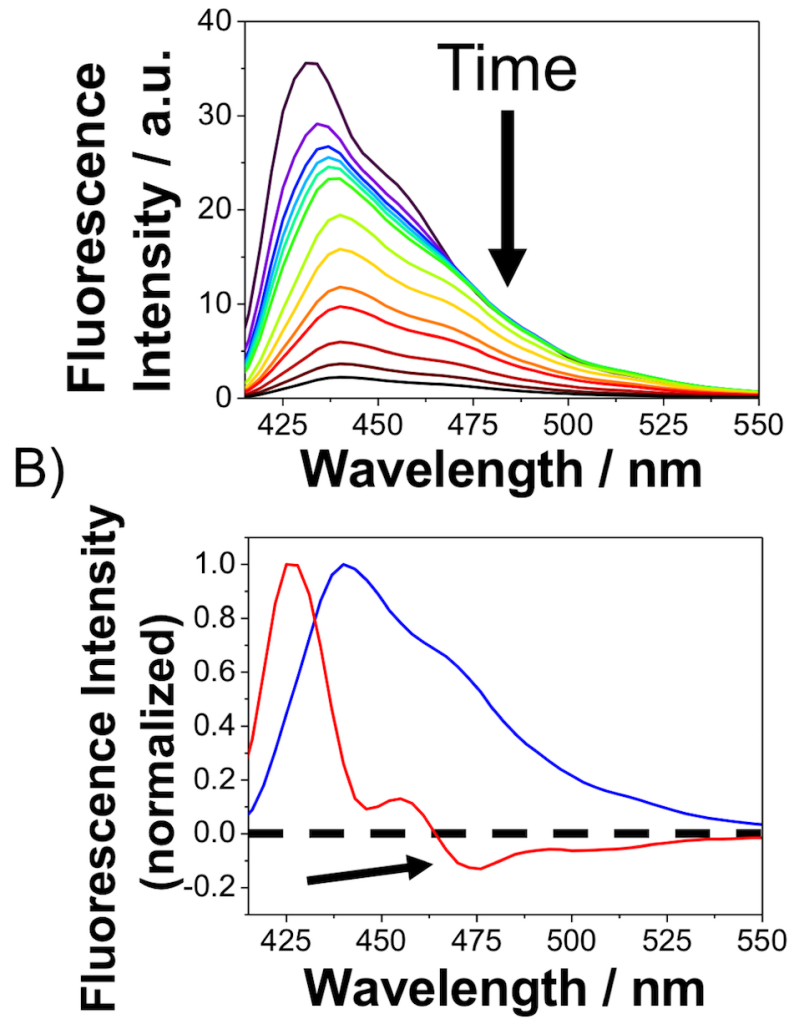

Figure 2. (A) TRES spectra of 1 dissolved in acetonitrile $\left(\lambda_{\mathrm{ex}}=375 \mathrm{~nm}\right)$ at $0,0.5,0.8,1,1.2,1.5,3,5,8,10,15,20$, and $25 \mathrm{~ns}$ after the excitation pulse. (B) SAEMS of $\mathbf{1}$ in acetonitrile, associated with the longest lifetime (blue) and the shortest lifetime (red).

$\mathrm{nm}$ in the initial $1.5 \mathrm{~ns}$, which indicated the excited-state transformation of the initially excited species into a different product. The species-associated emission spectra (SAEMS), as obtained from TRES measurements, represent the emission profiles linked to each one of the detected decay times. In a mixture of emissive dyes with different fluorescence lifetimes, the SAEMS would represent the spectrum of each form. However, in the presence of an excited-state reaction, the SAEMS provide information on the spectral distribution of the reagent, or directly excited species, and the final product arising during the excited-state reaction. ${ }^{17}$ The analysis of SAEMS clearly showed negative pre-exponential factors associated with the short decay time (rise time) from 460 to $500 \mathrm{~nm}$ (Figure $2 B$ ), indicating that the excited-state product has preferential emission in this spectral range. Similar results, confirming the formation of excimers, were found for acridone $\mathbf{1}$ dissolved in other solvents (Figures S3 and S4). The photophysical properties of acridone 1 responded mainly to microenviroment polarity, also including acidity or basicity of the solvent. ${ }^{19}$ In contrast, the emission features of the dye are almost invariable with other factors, such as $\mathrm{pH}$ (in the near-neutral range), ${ }^{14}$ viscosity (since the fluorophore does not have a rotorlike structure, see Figure S5), or the presence of typical biologically relevant species, such as metal ions, peroxide, sulfides, and sulfites (Figure S6).

DYNEX Imaging of Cellular Lipid Droplets. Next, we aimed to actively seek direct evidence of dynamic excimer formation of acridone $\mathbf{1}$ inside the fibroblast colon cell line CCD-18Co.

The formation of excited-state emissive species, such as an excimer, is unequivocally characterized by the appearance of short rise times in the emission kinetics, i.e., a negative preexponential factor associated with the short decay time in the fluorescence decay trace. ${ }^{14}$ This type of fluorophore dynamics has not yet been detected inside live cells. To do so, only timeresolved fluorimetry, working in single photon timing (SPT) mode, is capable of reliably detecting the rise time; hence, fluorescence lifetime imaging microscopy (FLIM) is the tool of choice. For the unprecedented detection of photophysical nanosecond kinetics of acridone $\mathbf{1}$ in live cells, we analyzed FLIM images, adjusting the fluorescence decays to a biexponential model. Importantly, we found specific subcellular areas in which the pre-exponential (amplitude) associated with the short decay time, termed as $\mathrm{A} 2$, was consistently negative (Figure 3A,B). This is an unequivocal feature of nanosecond excited-state dynamics, ${ }^{14,20}$ as explained earlier.

The DYNEX imaging approach consists of an image segmentation procedure, which selects only pixels with negative amplitude of the short decay time. When the DYNEX criterion was applied to the collected FLIM images of $\mathbf{1}$ in live cells, it highlighted specifically spherical organelles, whereas the dye that had spread through the cytosol and other organelles was mostly monomeric and was thus filtered out by DYNEX. Figure 3A shows the segmentation of lipid droplets using DYNEX, whereas Figure $3 \mathrm{~B}$ shows the distribution of amplitude values of the short decay time (A2) from the whole image in Figure 3A. Additional videos and animations illustrating the DYNEX segmentation method can be found as Supporting Videos S1-S4. The organelles selected by DYNEX imaging were morphologically spherical, and we identified them as lipid droplets through colocalization experiments with different organelle trackers (Figures 3C,D, S7-S10, and Table S2 in the SI). Therefore, using DYNEX, we were capable of specifically identifying lipid droplets within the cytoplasm.

It is important to note that for this approach to work properly, the long decay time of the fluorophore must be as large as possible. Hence, acridone derivatives are especially interesting candidates. $^{14}$ Nevertheless, among the different tested $\mathrm{N}$ substituted-4-methoxyacridone derivatives as intracellular probes, ${ }^{19}$ strikingly, we unequivocally detected intracellular dynamic excimer formation in acridone 1 bearing an $\mathrm{N}$-(3(benzyloxy)propyl) radical. We did not find evidence of dynamic excimers in live-cell imaging using $\mathrm{N}$-(3-(hydroxy)propyl)- or $\mathrm{N}$-(3-(methoxy)propyl)-4-methoxyacridones. Importantly, the fact that the detection of an acridone excimer in cellular lipid droplets is specific to dye $\mathbf{1}$ confirms that it is not an instrumental artifact due to detector jitter. We hypothesized that the higher hydrophobicity of the benzyl radical promoted accumulation of the dye in lipid droplets, thus facilitating the formation of the excimer. These results can be reconciled by comparing the lipophilicity of these three dyes. The consensus, simulated $\log P$ value (partition coefficient octanol/water) is 4.24 for acridone 1 , whereas it is 2.67 and 3.01 for $\mathrm{N}$-(3(hydroxy)propyl)- and $\mathrm{N}$-(3-(methoxy)propyl)-4-methoxyacridone, respectively, as obtained using the SwissADME tool. ${ }^{21}$ Therefore, the larger $\log P$ value of acridone 1 supports further 
A)

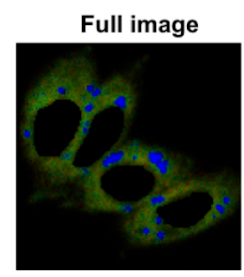

DYNEX segmentation A2 $<0$

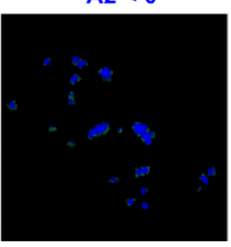

$\mathrm{A} 2>0$

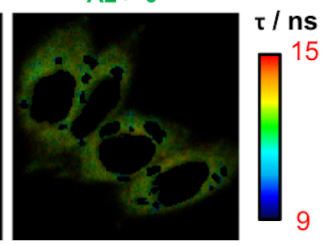

B)

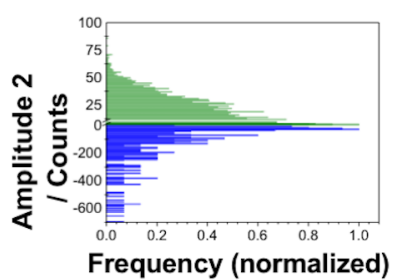

C)

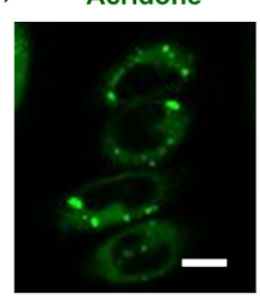

LipidTOX

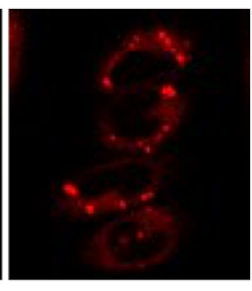

Overlay

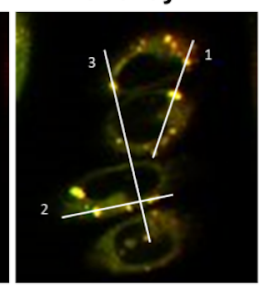

D)

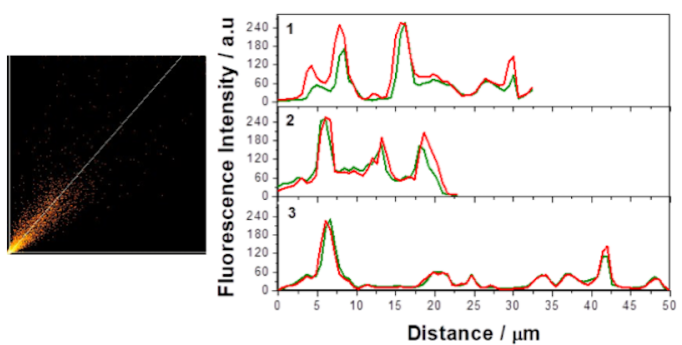

Figure 3. (A) FLIM image of dye 1 in CCD-18Co cells and segmentation procedure using DYNEX. Pixels with negative A2 amplitude are represented in blue. (B) Distribution of amplitudes of the short decay time (A2) of the image in panel (A). (C) Representative colocalization imaging of 1 (green channel) with LipidTOX (red channel). Scale bar of $10 \mu \mathrm{m}$. (D) Correlation plot (left) and intensity profiles of lines 1, 2, and 3 (right) from the green and red channels of panel (C).

accumulation in lipid droplets compared to the other studied dyes. The higher local concentration of the dye in these lipid droplets allows nanosecond, excited-state encounters between different molecules of the dye and, hence, the formation of actual dynamic excimers. Excimer formation, as a second-order reaction, is a concentration-dependent process. However, we did not find variations in the application of the DYNEX segmentation criterion with typical dye concentrations used for cell imaging in the sub- $\mu \mathrm{M}$ range. The negative amplitude values for A2 detected were high (Figure 3B), thus supporting that the methodology could be safely applied at much lower concentrations of dye 1 .

Next, we employed DYNEX imaging, combined with a validated multiparametric microscopy approach for determining microenvironment polarity, ${ }^{10}$ to compare the polarity inside lipid droplets in the human colon cell line CCD-18Co and the mouse colon carcinoma cell line CT26.WT. The polarity in lipid droplets is an indirect estimation of the lipid content and composition and can be related to altered metabolic states. ${ }^{22}$ For these measurements, once lipid droplet pixels were segmented using DYNEX, we extracted polarity $\mathrm{E}_{\mathrm{T}} 30$ values (Figure 4 ) via multiparametric microscopy by combining the time-gated intensity ratio and fluorescence lifetime information ${ }^{10}$ (see the SI for experimental details and Figures S11-S13). Heretofore, there have been some efforts to characterize intracellular polarity employing fluorescence microscopy techniques using emission spectra, ${ }^{23}$ fluorescence intensity, ${ }^{24}$ and ratiometric measures. ${ }^{25-27}$ Nevertheless, biological samples present important difficulties for quantitative imaging that are overcome by our methodology. ${ }^{10}$ Table 1 and Figure $\mathrm{S} 14$ show the $\mathrm{E}_{\mathrm{T}} 30$ values recovered from lipid droplets of CCD-18Co and CT26.WT cells. The average $\mathrm{E}_{\mathrm{T}} 30$ values were $46.1 \pm 0.1$ and $45.6 \pm 0.2$ $\mathrm{kcal} \mathrm{mol}^{-1}$ for CCD-18Co and CT26.WT cells, respectively. These values are consistent with droplets formed with neutral lipids surrounded by polar phospholipids. A close inspection of the droplet images (Figures 4B,C and S15) clearly exhibited lower polarity in the inner core of the droplets, in agreement with the aforementioned arrangement. ${ }^{26}$ Interestingly, compared with the CCD-18Co line, the tumoral CT26.WT cell line exhibited lipid droplets with slightly lower polarity, consistent with previous works. ${ }^{8,24,26-29}$ This observation supports the idea of a higher lipid content in the tumoral line due to altered, proliferative metabolism.

Although many different alternatives for fluorescently tagging lipid droplets are available, ${ }^{30}$ few probes are capable of quantitatively reporting lipid content and polarity. During the production phase of our work, several polarity probes specific for lipid droplets have been reported. ${ }^{31-33}$ In these recent works, the polarity of lipid droplets is quantified through conventional ratiometric imaging based on the spectral shift of solvatochromic probes. Klymchenko and colleagues further defined an arbitrary lipid order parameter to scale relative polarity changes, ${ }^{31}$ but absolute quantitative polarity values were not reported. In other cases, polarity changes in lipid droplets were only visualized but not quantified using fluorogenic reactions of solvatochromic probes. ${ }^{32}$ Interestingly, a coumarin-quinolinium dyad has been recently reported to respond to polarity changes using ratiometric and FLIM imaging. ${ }^{33}$ The response of this dye toward polarity was calibrated in terms of the dielectric constant, $\varepsilon$; however, when used in live cells, the detected intensity ratio values were out of the calibration values, making it impossible to quantify polarity. These recent reports illustrate the far superiority and advantages of our approach for in cellulo quantification of absolute polarity. First, DYNEX imaging allows specific filtering of pixels in lipid droplets without the need for an additional organelle tracker. A second important advantage of our method is that the simultaneous use of multidimensional information, lifetime, and time-gated intensity ratio provides robust and absolute determination of the polarity, in terms of the $\mathrm{E}_{\mathrm{T}} 30$ parameter, free from potential interferences from cellular autofluorescence and other sources of light, such as scatter. For comparison purposes, Table S3 in the SI gathers other fluorescent polarity probes for lipid droplets, the basis of the 
A)

Lipid droplets

\section{Cytosol}

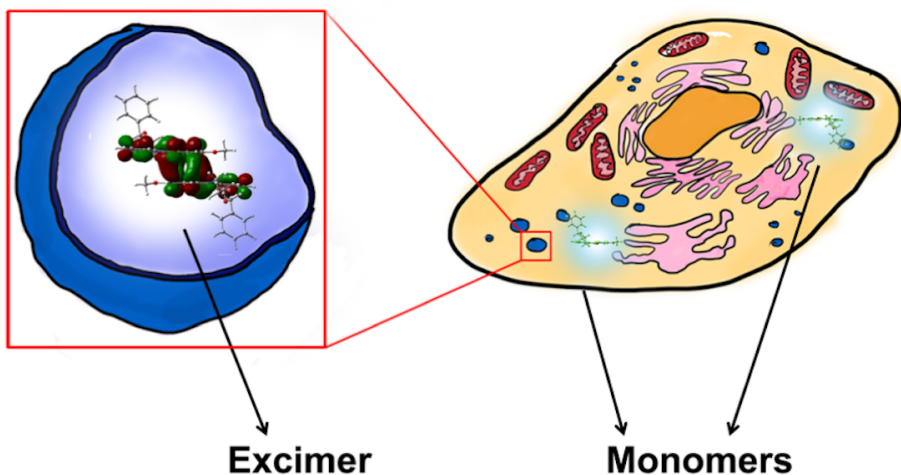

B)
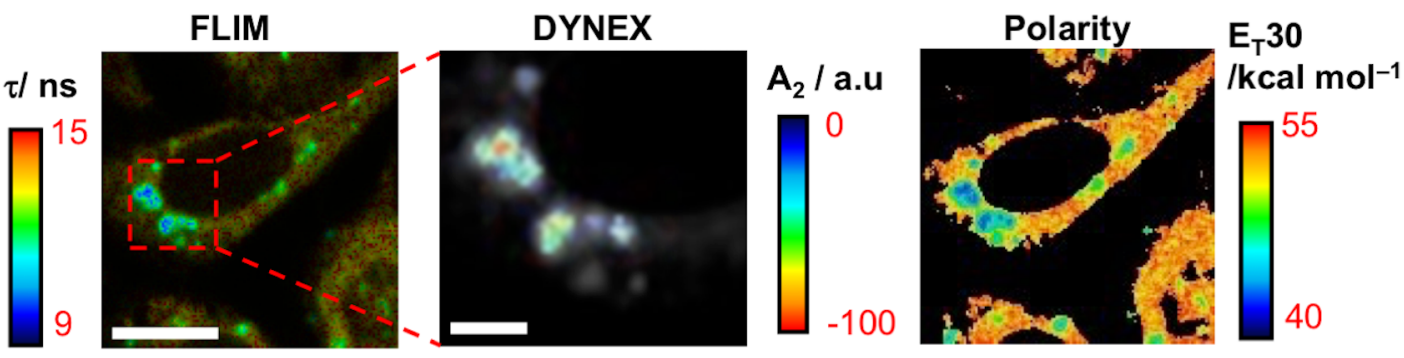

C)

FLIM

DYNEX
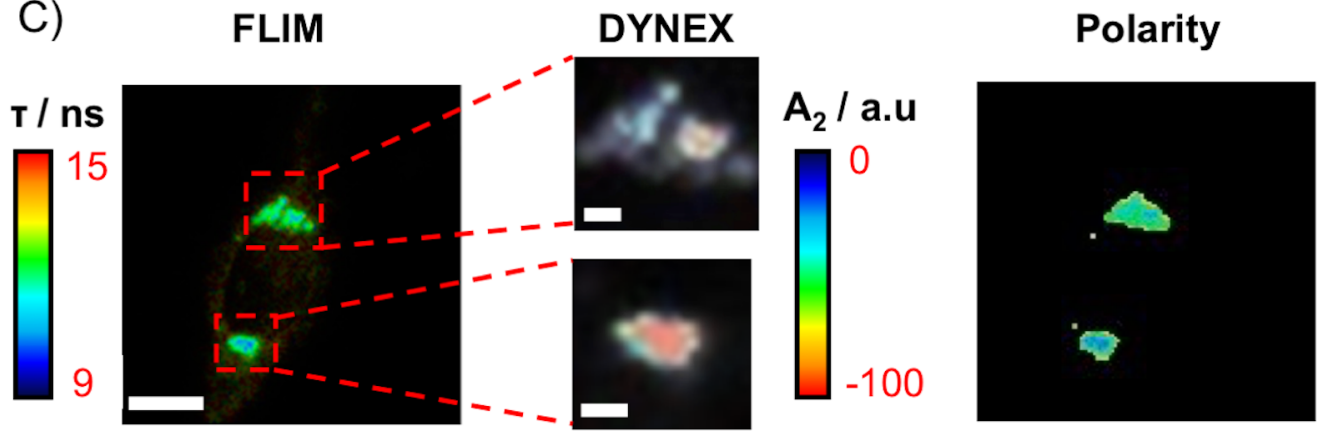

$\mathrm{E}_{\mathrm{T}} 30$

/kcal mol-1

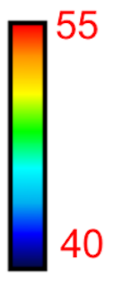

Figure 4. (A) Schematic of the DYNEX concept: selective formation of dynamic excimers of dye 1 in lipid droplets. (B, C) FLIM image (left panels), DYNEX segmentation of lipid droplets (central panels), and $\mathrm{E}_{\mathrm{T}} 30$ imaging (right panels) of 1 in CCD-18Co cells (B) and tumor CT26.WT cells (C). Scale bars represent $10 \mu \mathrm{m}$ for full images and $2 \mu \mathrm{m}$ for zoomed images.

\section{Table 1. $\mathrm{E}_{\mathrm{T}} 30$ Values in Lipid Droplets}

$\begin{array}{cllc}\text { cell line } & \text { treatment } & & \mathrm{E}_{\mathrm{T}} 30^{a}\left(\mathrm{kcal} \mathrm{mol}^{-1}\right) \\ \text { CCD-18Co } & & & 46.1 \pm 0.1 \\ & \text { sucrose } & 50 \mathrm{mM} & 46.8 \pm 0.1^{c} \\ & & 100 \mathrm{mM} & 47.1 \pm 0.2^{c} \\ \text { CT26.WT } & \text { chloroquine } & 25-100 \mu \mathrm{M} & 43.70 \pm 0.09^{d} \\ & & & 45.6 \pm 0.2^{e} \\ & \text { sucrose } & 50 \mathrm{mM} & 46.9 \pm 0.1^{c} \\ & & 100 \mathrm{mM} & 46.7 \pm 0.2^{c} \\ & \text { chloroquine } & 25-100 \mu \mathrm{M} & 42.3 \pm 0.1^{d}\end{array}$

${ }^{a}$ Errors are indicated as the standard error of the mean, s.e.m. ${ }^{b}$ Statistical comparison of populations was performed using the Mann-Whitney, Holm-Bonferroni, and Holm-Sidak statistical tests. ${ }^{c}$ Significantly different from the untreated control $(p<0.001)$. ${ }^{d}$ Significantly different from the untreated control $\left(p<10^{-25}\right)$. ${ }^{e}$ Significantly different from untreated CCD-18Co cells $(p<0.15)$.

imaging approach employed, and whether the sensor is capable of reporting absolute polarity values.
We further exploited the potential of DYNEX to measure how the polarity of lipid droplets was altered upon treatment with sucrose and chloroquine. Sucrose is known to increase membrane stability, interacting through hydroxyl groups and hence altering the polarity of intracellular compartments. ${ }^{34,35}$ On the other hand, chloroquine causes enhanced lipid production, potentially decreasing the polarity of lipid droplets. $^{22,34}$ After confirming that sucrose did not have any adverse effect on the photophysical properties of dye $\mathbf{1}$ (Figure S16), our results show that treatment with sucrose resulted in a significant increase in the polarity of lipid droplets $(p<0.001)$ for treatments with 50 and $100 \mathrm{mM}$ sucrose in both CCD-18Co and tumoral CT26.WT cells (Table 1, Figures 5, S17, and S18). Being a disaccharide, the internalization of sucrose strongly depends on the cellular type, usually running via endocytosis to lysosomes. For instance, the polarity of lysosomes has been shown to notably change upon sucrose treatment in MCF-7 breast cancer cells, ${ }^{34}$ but the effect was less noticeable in 293T cells and even lower in HepG2 cells, ${ }^{35}$ using $80 \mathrm{mM}$ sucrose in either case. The two cell lines that we used in our work are colon cells, with sufficient disaccharide receptors for endocytosis, and 
A)
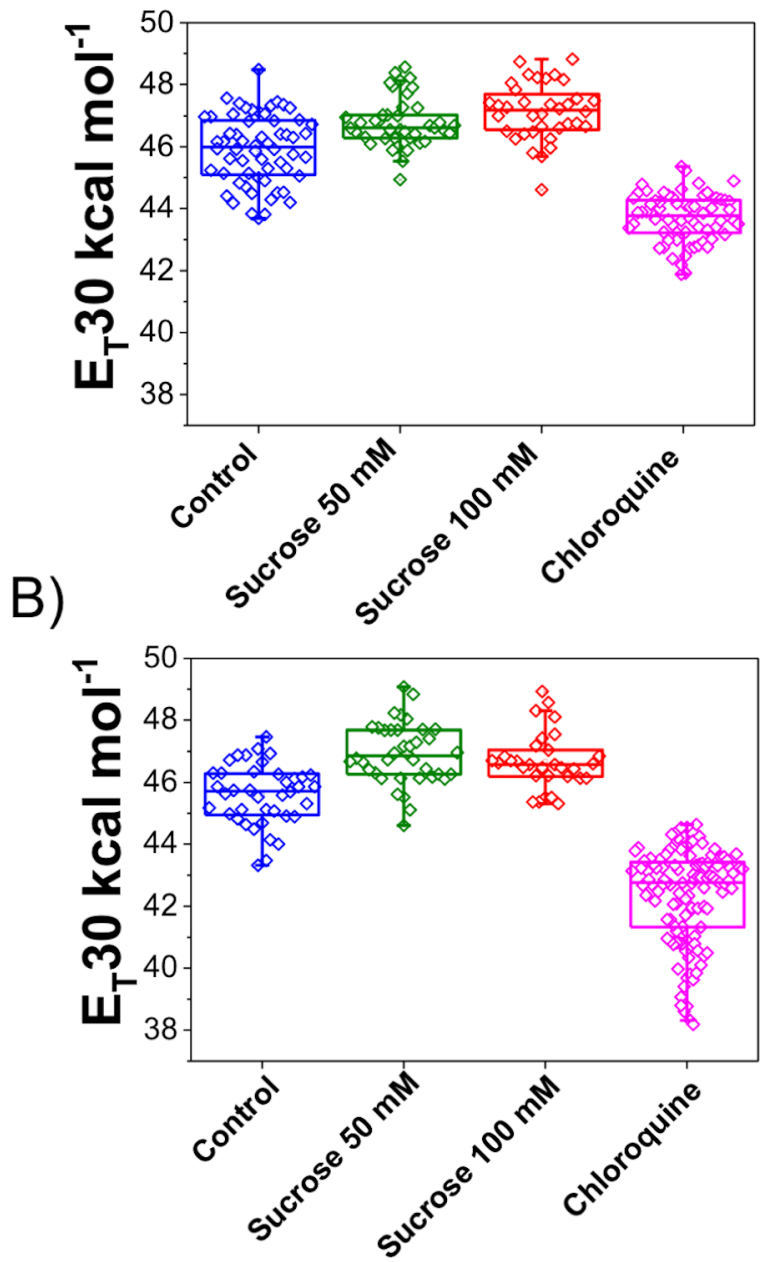

Figure 5. $\mathrm{E}_{\mathrm{T}} 30$ values in lipid droplets of CCD-18Co cells (A) and tumor CT26.WT cells (B) upon treatment with sucrose and chloroquine. Each individual value corresponds to lipid droplet regions from different cells from $>10$ different images.

the increase in polarity of lipid droplets that we have detected is then in line with previous results, considering the metabolic role of such organelles.

In contrast, treatment with chloroquine resulted in significantly more hydrophobic lipid droplets in both CCD-18Co and CT26.WT cell lines (Table 1, Figures 5 and S19). This result indicated a higher production of lipids stored in the corresponding organelles. ${ }^{22}$

\section{CONCLUSIONS}

In this work, we have demonstrated that segmentation by novel DYNEX imaging is a powerful approach for quantifying microenvironment polarity in cellular lipid droplets. The detection of an actual dynamic excimer inside live cells, as photophysically characterized by negative amplitudes on a decay time, i.e., a rise time, has not been previously reported. The fact that we detected a rise time is an unequivocal demonstration of such excited-state dynamics, as is well known in the photophysics field. ${ }^{14,36}$ For this advance, the extraordinary behavior of acridone dyes was a turning point in terms of both a well-defined excimer reaction, as evidenced in our computational and experimental studies, and a long decay time, leaving enough time, on the nanosecond timescale, to detect the formation of excited-state interactions using FLIM microscopy in SPT mode. In this design, the solvatochromism of the acridone core is able to resolve the microenvironment polarity, while the $\mathrm{N}$ substituent allows convenient placement of the probe in the correct organelle and facilitates excimer formation.

In the past, changes in fluorescence associated with the formation of excimers, such as quenching of monomer emission or red-shifted excimer emission, have been exploited for some time in imaging for sensing purposes, ${ }^{37-40}$ for instance, of metallic ions, such as $\mathrm{Al}^{3+38} \mathrm{Zn}^{2+}, 40$ and $\mathrm{Hg}^{2+39}$ and biomolecules, such as heparin, ${ }^{37}$ cysteine, $^{40}$ and phosphate. ${ }^{40}$ Most of these works employed common dyes with well-known excimer emission, such as pyrene ${ }^{38-40}$ or perylene. ${ }^{37}$ However, all of these works were based on the usual red-shifted emission of the excimers and used ratiometric intensity approaches ${ }^{37,38}$ or off/on behavior ${ }^{39,40}$ to illustrate the detection of a different emissive species. Since these works used steady-state intensity, one cannot provide kinetic information on the dynamics of the dye nor unequivocally confirm whether the red-shifted emissive species was dynamically formed in the excited state (an actual excimer) or was preformed in the ground state (static aggregates). The detection of red-shifted, enhanced emission upon dimerization or aggregation of certain dyes is well known and has given rise to widespread studies on aggregation-induced emission (AIE). ${ }^{41}$ To the best of our knowledge, direct measurement of environment-dependent excited-state excimer kinetics inside live cells has not been previously employed in fluorescence imaging. Therefore, our observations are important not only from the point of view of the photophysics of a dye detected intracellularly but also because they allowed us to develop a novel segmentation procedure via DYNEX imaging, an approach that exploits the use of time-resolved excited-state kinetic information to gain a new layer for image filtering in fluorescence microscopy, complementary to other common fluorescence parameters.

In this regard, similar methodologies of imaging analysis and filtering based on excited-state kinetics of the fluorescent probe can be foreseen. In the past, we successfully developed fluorescent sensors for phosphate imaging, based on excitedstate proton transfer reactions of xanthene derivatives. ${ }^{42,43}$ Therefore, we envisage that the appropriate combination of rich dye's photophysics and time-resolved fluorescence imaging tools, such as FLIM microscopy, will pave the way for the design of new multidimensional fluorescent sensors able to provide target-specific and orthogonal information, including spectral, intensity, lifetime, and excited-state kinetic changes, among others.

\section{ASSOCIATED CONTENT}

\section{S1 Supporting Information}

The Supporting Information is available free of charge at https://pubs.acs.org/doi/10.1021/acssensors.1c01206.

Detailed materials and methods; supporting results from quantum chemistry calculations (Chart S1, Figure S1, and Table S1); supporting spectroscopic results (Figures S2S6); subcellular localization study (Figures S7-S10 and Table S2); additional DYNEX images and results (Figures S11-S15); comparison of different lipid droplet polarity probes (Table S3); and sucrose and chloroquine treatments (Figures S16-S19) (PDF)

Supporting Videos S1-S4 illustrating the DYNEX segmentation method (ZIP) 


\section{AUTHOR INFORMATION}

\section{Corresponding Authors}

Emilio Garcia-Fernandez - Departamento de Fisicoquimica, Unidad de Excelencia en Quimica Aplicada a Biomedicina y Medioambiente (UEQ), Facultad de Farmacia, Universidad de Granada, 18071 Granada, Spain; Email: emiliogf@ugr.es Angel Orte - Departamento de Fisicoquimica, Unidad de Excelencia en Quimica Aplicada a Biomedicina y Medioambiente (UEQ), Facultad de Farmacia, Universidad de Granada, 18071 Granada, Spain; $\odot$ orcid.org/00000003-1905-4183; Email: angelort@ugr.es

\section{Authors}

M. Carmen Gonzalez-Garcia - Departamento de Fisicoquimica, Unidad de Excelencia en Quimica Aplicada a Biomedicina y Medioambiente (UEQ), Facultad de Farmacia, Universidad de Granada, 18071 Granada, Spain

Carmen Salto-Giron - Departamento de Fisicoquimica, Unidad de Excelencia en Quimica Aplicada a Biomedicina y Medioambiente (UEQ), Facultad de Farmacia, Universidad de Granada, 18071 Granada, Spain

Pilar Herrero-Foncubierta - Departamento de Fisicoquimica, Unidad de Excelencia en Quimica Aplicada a Biomedicina y Medioambiente (UEQ), Facultad de Farmacia, Universidad de Granada, 18071 Granada, Spain; Departamento de Quimica Organica, Unidad de Excelencia en Quimica Aplicada a Biomedicina y Medioambiente (UEQ), Facultad de Ciencias, Universidad de Granada, 18071 Granada, Spain

Tomás Peña-Ruiz - Departamento de Química Física y Analitica, Facultad de Ciencias Experimentales, Universidad de Jaén, 23071 Jaén, Spain

Maria Dolores Giron-Gonzalez - Departamento de Bioquimica y Biologia Molecular II, Unidad de Excelencia en Quimica Aplicada a Biomedicina y Medioambiente (UEQ), Facultad de Farmacia, Universidad de Granada, 18071 Granada, Spain; ๑ orcid.org/0000-0001-9638-988X

Rafael Salto-Gonzalez - Departamento de Bioquimica y Biologia Molecular II, Unidad de Excelencia en Quimica Aplicada a Biomedicina y Medioambiente (UEQ), Facultad de Farmacia, Universidad de Granada, 18071 Granada, Spain; (1) orcid.org/0000-0002-7044-3611

Angel Perez-Lara - Departamento de Fisicoquimica, Unidad de Excelencia en Quimica Aplicada a Biomedicina y Medioambiente (UEQ), Facultad de Farmacia, Universidad de Granada, 18071 Granada, Spain; Department of Neurobiology, Max Planck Institute for Biophysical Chemistry, 37077 Göttingen, Germany

Amparo Navarro - Departamento de Química Física y Analítica, Facultad de Ciencias Experimentales, Universidad de Jaén, 23071 Jaén, Spain; @ orcid.org/0000-0001-96206668

Complete contact information is available at: https://pubs.acs.org/10.1021/acssensors.1c01206

\section{Author Contributions}

The manuscript was written through contributions of all authors. All authors have given approval to the final version of the manuscript.

\section{Notes}

The authors declare no competing financial interest.

\section{ACKNOWLEDGMENTS}

This work was funded by grants CTQ2017-85658-R (MICIU/ AEI/ERDF), FQM-337 (Junta de Andalucía), and PIUJA 201920 (Universidad de Jaén). We acknowledge the Universidad de Granada (Spain) microscopy central facilities (CIC-UGR) and computing time from CSIRC-UGR. M.C.G.-G. thanks MICIU/ AEI for a predoctoral fellowship. Funding for open access charge: Universidad de Granada/CBUA. We are indebted to Prof. Reinhard Jahn for his generous support.

\section{ABBREVIATIONS}

DYNEX, dynamic excimer imaging; TD-DFT, time-dependent density functional theory; FLIM, fluorescence lifetime imaging microscopy; TRES, time-resolved emission spectroscopy; SAEMS, species-associated emission spectra

\section{REFERENCES}

(1) Yang, Z.; Cao, J.; He, Y.; Yang, J. H.; Kim, T.; Peng, X.; Kim, J. S. Macro-/micro-environment-sensitive chemosensing and biological imaging. Chem. Soc. Rev. 2014, 43, 4563-4601.

(2) Olzmann, J. A.; Carvalho, P. Dynamics and functions of lipid droplets. Nat. Rev. Mol. Cell Biol. 2019, 20, 137-155.

(3) Welte, M. A. Expanding Roles for Lipid Droplets. Curr. Biol. 2015, 25, R470-R481.

(4) Park, S. J.; Juvekar, V.; Jo, J. H.; Kim, H. M. Combining hydrophilic and hydrophobic environment sensitive dyes to detect a wide range of cellular polarity. Chem. Sci. 2020, 11, 596-601.

(5) Ashoka, A. H.; Ashokkumar, P.; Kovtun, Y. P.; Klymchenko, A. S. Solvatochromic Near-Infrared Probe for Polarity Mapping of Biomembranes and Lipid Droplets in Cells under Stress. J. Phys. Chem. Lett. 2019, 10, 2414-2421.

(6) Yin, J.; Peng, M.; Ma, Y.; Guo, R.; Lin, W. Rational design of a lipid-droplet-polarity based fluorescent probe for potential cancer diagnosis. Chem. Commun. 2018, 54, 12093-12096.

(7) Collot, M.; Fam, T. K.; Ashokkumar, P.; Faklaris, O.; Galli, T.; Danglot, L.; Klymchenko, A. S. Ultrabright and Fluorogenic Probes for Multicolor Imaging and Tracking of Lipid Droplets in Cells and Tissues. J. Am. Chem. Soc. 2018, 140, 5401-5411.

(8) Ghosh, C.; Nandi, S.; Bhattacharyya, K. Probing microenvironment of lipid droplets in a live breast cell: MCF7 and MCF10A. Chem. Phys. Lett. 2017, 670, 27-31.

(9) Lu, B.; Yin, J.; Liu, C.; Lin, W. Lipid droplet polarity decreases during the pathology of muscle injury as revealed by a polarity sensitive sensor. Spectrochim. Acta, Part A 2021, 262, No. 120149.

(10) Gonzalez-Garcia, M. C.; Herrero-Foncubierta, P.; GarciaFernandez, E.; Orte, A. Building Accurate Intracellular Polarity Maps through Multiparametric Microscopy. Methods Protoc. 2020, 3, No. 78.

(11) Cerón-Carrasco, J. P.; Jacquemin, D.; Laurence, C.; Planchat, A.; Reichardt, C.; Sraïdi, K. Solvent polarity scales: determination of new ET(30) values for 84 organic solvents. J. Phys. Org. Chem. 2014, 27, $512-518$.

(12) Garcia-Fernandez, E.; Pernagallo, S.; González-Vera, J. A.; Ruedas-Rama, M. J.; Díaz-Mochón, J. J.; Orte, A. Time-Gated Luminescence Acquisition for Biochemical Sensing: miRNA Detection. In Fluorescence in Industry; Pedras, B., Ed.; Springer International Publishing: Cham, 2019; Vol. 18, pp 213-267.

(13) Herrero-Foncubierta, P.; González-García, M. D. C.; Resa, S.; Paredes, J. M.; Ripoll, C.; Girón, M. D.; Salto, R.; Cuerva, J. M.; Orte, A.; Miguel, D. Simple and non-charged long-lived fluorescent intracellular organelle trackers. Dyes Pigm. 2020, 183, No. 108649.

(14) Gonzalez-Garcia, M. C.; Herrero-Foncubierta, P.; Castro, S.; Resa, S.; Alvarez-Pez, J. M.; Miguel, D.; Cuerva, J. M.; GarciaFernandez, E.; Orte, A. Coupled Excited-State Dynamics in NSubstituted 2-Methoxy-9-Acridones. Front. Chem. 2019, 7, No. 129.

(15) Grimme, S.; Antony, J.; Ehrlich, S.; Krieg, H. A consistent and accurate $\mathrm{ab}$ initio parametrization of density functional dispersion 
correction (DFT-D) for the 94 elements H-Pu. J. Chem. Phys. 2010, 132, No. 154104.

(16) Kołaski, M.; Arunkumar, C. R.; Kim, K. S. Aromatic Excimers: Ab Initio and TD-DFT Study. J. Chem. Theory Comput. 2013, 9, 847-856.

(17) Lakowicz, J. R. Principles of Fluorescence Spectroscopy, 3rd ed.; Springer: New York, 2006.

(18) Crovetto, L.; Orte, A.; Paredes, J. M.; Resa, S.; Valverde, J.; Castello, F.; Miguel, D.; Cuerva, J. M.; Talavera, E. M.; Alvarez-Pez, J. M. Photophysics of a Live-Cell-Marker, Red Silicon-Substituted Xanthene Dye. J. Phys. Chem. A 2015, 119, 10854-10862.

(19) Gonzalez-Garcia, M. C.; Peña-Ruiz, T.; Herrero-Foncubierta, P.; Miguel, D.; Giron, M. D.; Salto, R.; Cuerva, J. M.; Navarro, A.; GarciaFernandez, E.; Orte, A. Orthogonal cell polarity imaging by multiparametric fluorescence microscopy. Sens. Actuators, B 2020, 309, No. 127770.

(20) Orte, A.; Bermejo, R.; Talavera, E. M.; Crovetto, L.; Alvarez-Pez, J. M. 2', $7^{\prime}$-Difluorofluorescein Excited-State Proton Reactions: Correlation between Time-Resolved Emission and Steady-State Fluorescence Intensity. J. Phys. Chem. A 2005, 109, 2840-2846.

(21) Daina, A.; Michielin, O.; Zoete, V. SwissADME: a free web tool to evaluate pharmacokinetics, drug-likeness and medicinal chemistry friendliness of small molecules. Sci. Rep. 2017, 7, No. 42717.

(22) Bik, E.; Mateuszuk, L.; Orleanska, J.; Baranska, M.; Chlopicki, S.; Majzner, K. Chloroquine-Induced Accumulation of Autophagosomes and Lipids in the Endothelium. Int. J. Mol. Sci. 2021, 22, No. 2401.

(23) Pal, K.; Kumar, P.; Koner, A. L. Deciphering interior polarity of lysosome in live cancer and normal cells using spectral scanning microscopy. J. Photochem. Photobiol., B 2020, 206, No. 111848.

(24) Gai, F.; Zuo, Y.; Lin, W. Detecting lipid droplets polarity: Silicone-based unique fluorescent probe for cancer diagnosis in living cells. Talanta 2021, 225, No. 122059.

(25) Danylchuk, D. I.; Jouard, P.-H.; Klymchenko, A. S. Targeted Solvatochromic Fluorescent Probes for Imaging Lipid Order in Organelles under Oxidative and Mechanical Stress. J. Am. Chem. Soc. 2021, 143, 912-924.

(26) Collot, M.; Bou, S.; Fam, T. K.; Richert, L.; Mély, Y.; Danglot, L.; Klymchenko, A. S. Probing Polarity and Heterogeneity of Lipid Droplets in Live Cells Using a Push-Pull Fluorophore. Anal. Chem. 2019, 91, 1928-1935.

(27) Dai, Y.; Zhan, Z.; Li, Q.; Liu, R.; Lv, Y. Simultaneous monitoring of polarity changes of lipid droplets and lysosomes with two-photon fluorescent probes. Anal. Chim. Acta 2020, 1136, 34-41.

(28) Yin, J.; Peng, M.; Lin, W. Two-photon fluorescence imaging of lipid drops polarity toward cancer diagnosis in living cells and tissue. Sens. Actuators, B 2019, 288, 251-258.

(29) Chowdhury, R.; Jana, B.; Saha, A.; Ghosh, S.; Bhattacharyya, K. Confocal microscopy of cytoplasmic lipid droplets in a live cancer cell: number, polarity, diffusion and solvation dynamics. MedChemComm 2014, 5, 536-539.

(30) Fam, T. K.; Klymchenko, A. S.; Collot, M. Recent Advances in Fluorescent Probes for Lipid Droplets. Materials 2018, 11, No. 1768.

(31) Danylchuk, D. I.; Jouard, P.-H.; Klymchenko, A. S. Targeted Solvatochromic Fluorescent Probes for Imaging Lipid Order in Organelles under Oxidative and Mechanical Stress. J. Am. Chem. Soc. 2021, 143, 912-924.

(32) Fan, L.; Wang, X.; Zan, Q.; Fan, L.; Li, F.; Yang, Y.; Zhang, C.; Shuang, S.; Dong, C. Lipid Droplet-Specific Fluorescent Probe for In Vivo Visualization of Polarity in Fatty Liver, Inflammation, and Cancer Models. Anal. Chem. 2021, 93, 8019-8026.

(33) Wang, K.-N.; Liu, L.-Y.; Mao, D.; Xu, S.; Tan, C.-P.; Cao, Q.; Mao, Z.-W.; Liu, B. A Polarity-Sensitive Ratiometric Fluorescence Probe for Monitoring Changes in Lipid Droplets and Nucleus during Ferroptosis. Angew. Chem., Int. Ed. 2021, 60, 15095-15100.

(34) Zhu, H.; Fan, J.; Mu, H.; Zhu, T.; Zhang, Z.; Du, J.; Peng, X. dPET-controlled "off-on" Polarity-sensitive Probes for Reporting Local Hydrophilicity within Lysosomes. Sci. Rep. 2016, 6, No. 35627.

(35) Hu, L.; Shi, D.; Li, X.; Zhu, J.; Mao, F.; Li, X.; Xia, C.; Jiang, B.; Guo, Y.; Li, J. Curcumin-based polarity fluorescent probes: Design strategy and biological applications. Dyes Pigm. 2020, 177, No. 108320.
(36) Orte, A.; Crovetto, L.; Talavera, E. M.; Boens, N.; Alvarez-Pez, J. M. Absorption and Emission Study of $2^{\prime}, 7^{\prime}$-Difluorofluorescein and Its Excited-State Buffer-Mediated Proton Exchange Reactions. J. Phys. Chem. A 2005, 109, 734-747.

(37) Li, J.; Xu, J.; Guo, W.; Zhong, W.; Li, Q.; Tan, L.; Shang, L. Ratiometric fluorescence sensors for heparin and heparinase based on enhanced excimer emission of perylene probe induced by cationic silver nanoparticles. Sens. Actuators, B 2020, 305, No. 127422.

(38) Das, S.; Sahana, A.; Banerjee, A.; Lohar, S.; Safin, D. A.; Babashkina, M. G.; Bolte, M.; Garcia, Y.; Hauli, I.; Mukhopadhyay, S. K.; Das, D. Ratiometric fluorescence sensing and intracellular imaging of $\mathrm{Al}^{3+}$ ions driven by an intramolecular excimer formation of a pyrimidine-pyrene scaffold. Dalton Trans. 2013, 42, 4757-4763.

(39) Razi, S. S.; Ali, R.; Srivastava, P.; Misra, A. Smart excimer fluorescence probe for visual detection, cell imaging and extraction of $\mathrm{Hg}^{2+}$. RSC Adv. 2015, 5, 79538-79547.

(40) Upadhyay, Y.; Anand, T.; Babu, L. T.; Paira, P.; Crisponi, G.; Sk, A. K.; Kumar, R.; Sahoo, S. K. Three-in-one type fluorescent sensor based on a pyrene pyridoxal cascade for the selective detection of $\mathrm{Zn}$ (II), hydrogen phosphate and cysteine. Dalton Trans. 2018, 47, $742-749$.

(41) Gao, M.; Tang, B. Z. Fluorescent Sensors Based on AggregationInduced Emission: Recent Advances and Perspectives. ACS Sens. 2017, 2, 1382-1399.

(42) Zhang, Q.; Xiao, K.; Paredes, J. M.; Mamonova, T.; Sneddon, W. B.; Liu, H.; Wang, D.; Li, S.; McGarvey, J. C.; Uehling, D.; Al-awar, R.; Joseph, B.; Jean-Alphonse, F.; Orte, A.; Friedman, P. A. Parathyroid hormone initiates dynamic NHERF1 phosphorylation cycling and conformational changes that regulate NPT2A-dependent phosphate transport. J. Biol. Chem. 2019, 294, 4546-4571.

(43) Paredes, J. M.; Giron, M. D.; Ruedas-Rama, M. J.; Orte, A.; Crovetto, L.; Talavera, E. M.; Salto, R.; Alvarez-Pez, J. M. Real-Time Phosphate Sensing in Living Cells using Fluorescence Lifetime Imaging Microscopy (FLIM). J. Phys. Chem. B 2013, 117, 8143-8149. 